\title{
Surgical therapy for herlyn-werner-wunderlich (HWW) syndrome cases
}

\begin{abstract}
Introduction: One of the rare forms of Mullerian congenital ducts is Herlyn-WernerWunderlich (HWW) syndrome. HWW syndrome is usually found at puberty with nonspecific symptoms. Pelvic pain can be found both acute and chronic, dysmenorrhoea, as well as hematometra and hematocolpos. Early detection and treatment results in a good prognosis with fertility preservation.
\end{abstract}

Case presentation: Two cases of Herlyn-Werner-Wunderlich (HWW) syndrome surgically performed were reported at Dr. Sardjito Yogyakarta Indonesia Central General Hospital in 2017-2018. Both cases are still children and at the age of puberty. The chief complaint in both cases it was reported to have the same main complaint, abdominal pain, with the condition having never experienced menstruation yet. On further examination it is known that pain is caused due to hematometra and hematosalping that occur in both cases. The first case with hematometra in the right hemiuterus and right hematosalpinx, while second case with hematometra in the uterus didelphys and bilateral hematosalpinx. One case with a normal vagina, and the other case of vaginal agenesis. There was previous history of surgery in both of cases. There are abnormalities in the urinary tract that occur together with these genital abnormalities. Surgical therapy is carried out with the main goal being to drain menstrual blood from hematometra and hematosalpinx. In first case which has a vagina within the normal range $(9 \mathrm{~cm}$ long) and the inferior area of the right hemiuterus which is bulging toward the vagina, an area can be identified for the drainage incision to drain the entire hematometra and hematosalpinx. In the second case with vaginal agenesis, neovagina which is made by sigmoid colon vaginoplasty surgery can be a way of menstrual blood flow for a lifetime.

Conclusion: Clinical manifestations of Herlyn-Werner-Wunderlich (HWW) syndrome cases can be classified into 2 groups, complete and incomplete. Surgical treatment is based on the condition of the uterus and vagina. In the condition of cervical agenesis but with a normal vagina, surgery can be done by making an incision in the drainage of the uterine cervical tissue. If vaginal agenesis is obtained, vaginoplasty surgery can be performed, and one of the options is sigmoid colon vaginoplasty.

Keywords: congenital anomaly, Herlyn-Werner-Wunderlich (HWW) syndrome, vaginal agenesis, renal agenesis

\author{
Volume 5 Issue 5 - 202I
}

\begin{abstract}
Pangastuti N,' Rahman MN,' Setyawan $N^{2}$
'Department of Obstetrics and Gynaecology, Faculty of Medicine, Public Health and Nursing, Universitas Gadjah MadaSardjito Hospital, Yogyakarta, Indonesia

${ }^{2}$ Department of Surgery, Faculty of Medicine, Public Health and Nursing, Universitas Gadjah Mada-Sardjito Hospital,Yogyakarta, Indonesia
\end{abstract}

\begin{abstract}
Correspondence: Pangastuti N, Department of Obstetrics and Gynaecology, Faculty of Medicine, Public Health and Nursing, Universitas Gadjah Mada-Sardjito Hospital,Yogyakarta, Indonesia, Tel +628I22703752, Email nuring_nw@yahoo.co.id
\end{abstract}

Received: September 28, 202I | Published: October 22, 2021

\section{Introduction}

The female reproductive tract consisting of the tube, uterus, cervix, and upper part of the vagina, comes from the Mullerian duct (paramesonephric). Mullerian duct that does not develop or experiences defects can result in agenesis or hypoplasia, vertical or lateral fusion defects. The appearing disorders include hypoplasia or agenesis, uterine didelphys, unicornuate or bicornuate uterus, vaginal anomalies such as agenesis or duplication. ${ }^{1-3}$

A rare form of the congenital abnormality of the Mullerian duct is Herlyn-Werner-Wunderlich (HWW) syndrome. This syndrome occurs as a result of the failure of the lateral and vertical fusion of the Mullerian duct at 9 weeks' gestation.

The manifestations of abnormalities are didelphys uterus, hemivagina with obstruction, and ipsilateral renal agenesis. ${ }^{4}$ Ipsilateral Mullerian duct anomalies that have obstruction greater than $50 \%$ are actually predictive factors for renal agenesis. This anomaly is dominant, twice as often on the right side. With the existence of several relationships between the anomalies of the urogenital organ and the female genitalia, the urogenital system should be examined in the case of female genital organ anomalies. ${ }^{1,2}$
HWW syndrome is usually known after menarche because of complaints of abdominal pain due to hematocolpos or dysmenorrhoea. This condition can be treated with surgery. Early diagnosis will provide benefits because late diagnosis can cause several complications, like retrograde tubal reflux, leading to endometriosis and infertility. ${ }^{4}$

Lan Zhu et al. looked at patients with HWW syndrome at Peking Medical College Hospital (PUMCH), and suggested a new classification for HWW syndrome, based on the presence or absence of complete or incomplete vaginal obstruction. This syndrome is classified by classification 1 (complete obstruction hemivagina) and classification 2 (incomplete hemivaginal obstruction). In classification 2 in which hemivaginal obstruction is incomplete, the menstrual process can run normally from the side that is not obstructed, so it does not always cause problems or complaints and cause delays in diagnosis Figure 1.,5

Other anomalies that can be found are renal dysplasia, kidney and ureter duplication, ectopic ureter, high aortic bifurcation, duplication of IVC, intestinal malrotation and ovarian malposition. Renal agenesis is ipsilateral to the dilated uterine cavity. The right side is twice more affected than the left side. ${ }^{3,7}$ 


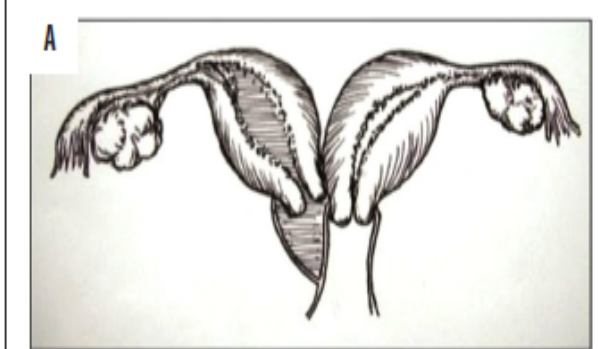

B

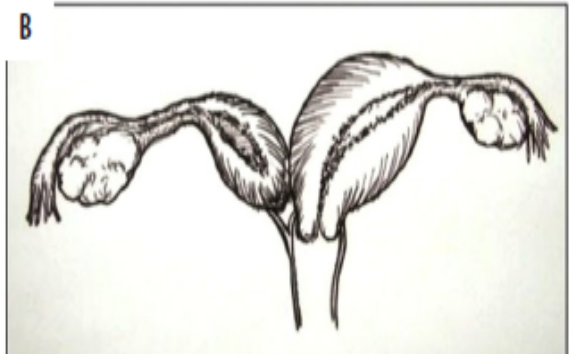

Figure 6. Classification 1 - Completely obstructed hemivagina. Classification 1.1 - with blind hemivagina (A). There is no communication between the duplicated uterus and vagina. Haematocolpos occurs a few months after menarche. Haemoperitoneum due to bleeding from the fallopian tube can be found at surgery [10]. If neglected, the condition can progress to secondary endometriosis, pelvic adhesions, pyosalpinx and pyocolpos [10,11]. Classification 1.2 - Cervicovaginal atresia without communicating uteri (B). Cervix behind the septum is atretic or maldeveloped. Menses from the uterus behind the septum cannot flow through atretic cervix. Hence, dinical features are similar to as in classification 1.1. Classification 2 - Incompletely obstructed hemivagina. Classification 2.1 - Partial reabsorption of the vaginal septum (C). A small communication exists between two vaginas with resultant incomplete obstruction. Patients have a late presentation. Classification 2.2, with communicating uteri (D). A small communication exists between two duplicated cervices with a completely obstructed hemivagina. Menses from the uterus behind the septum can flow through communication to the contralateral cervix. Drainage is often impeded due to small communication. Courtesy: Zhu etal. New classification of Herlyn-Werner-Wunderlich syndrome. Chin Med J, 2015; 128:222-25.

Figure I A new classification for HWW syndrome. ${ }^{5,6}$

HWW syndrome is usually found at puberty with nonspecific symptoms. Pelvic pain can be found both in acute and chronic conditions, dysmenorrhea, and the presence of hematometra or hematocolpos. Giving analgesics can reduce and even kill the pain of the patients. As a result, it often blurs the diagnosis. Complications can occur in the form of pyohematocolpos or pyosalpinx, as well as pelvic peritonitis. Long-term complications are endometriosis, pelvic adhesion which causes chronic pelvic pain and infertility.

The use of supporting examination tools such as ultrasonography (USG) and MRI has an important role in detecting HWW syndrome. Transvaginal sonography has the advantage of imaging the uterus and adnexa more clearly. It costs less, produces better results and does not involve radiation exposure. MRI is considered the gold standard for the pre-operative diagnosis and planning of Herlyn-WernerWunderlich syndrome.

Early detection and treatment produce a good prognosis with fertility preservation. As many as $80 \%$ of patients with didelphis uterus who are pregnant have an increase in the rate of preterm birth $(22 \%)$ and abortion ( $74 \%)$. Caesarean section is needed in more than $80 \%$ of patients. A rare complication is adenocarcinoma from the side of the uterine cervix that has obstruction and clear cell carcinoma of the obstructed vagina. ${ }^{5}$

This report is a retrospective case study of two patients with vaginal agenesis, who were performed surgically at Dr. Sardjito Hospital Yogyakarta in 2017-2018. Data taken from medical records includes general patient data, medical history, surgical procedures, and final results. The surgical procedure is performed via laparotomy and vaginal, in collaboration with urologists and gastrointestinal surgeons. Identification of the anatomical structure of the reproductive organs was carried out, both patients had menstrual uterine function.

\section{Case presentation}

\section{Case I}

Case 1 was a 10-year-old P0A0, with a history of laparotomy for drainage of the right side of the hematometra and right-sided hematosalping, at 1,5 years before coming to Sardjito Hospital. The main complaint of the patient is abdominal pain, the patient has never had a period.

Based on the examination data obtained abdomen flat, supple, painless, there is a scar in the median line from the previous surgery. Urethra and vulva are within normal limits, there is a vaginal introitus, the hymen is intact, does not appear bulging, with a sonde can be checked the depth of the vaginal canal on the left lateral side $9 \mathrm{~cm}$. From the rectal examination, it is found that the uterus is enlarged on the right side, without pain. From abdominal ultrasound, uterine size data were obtained $10 \times 8 \times 7,8 \mathrm{~cm} 3$, the right side hematosalping was $3,65 \times 4,62 \times 4 \mathrm{~cm} 3$, the left kidney was visible, the right kidney was not visible. Abdominal radiograph and intravenous pyelography obtained non-visual right kidney, agenesis of right kidney, left kidney anatomy and function within normal limits, left ureter within normal limits, bladder function and bowel movements within normal limits, no opaque stones appeared in the channel left urinary, visualized 
bone system. From the colon in loop examination, there are no visible infiltrations, indentations, and signs of colitis in the visualized colorectal system.

At the time of the surgery, the following data were obtained. The uterus is a hemiuterus on the right side with size of $10 \times 8 \times 8 \mathrm{~cm} 3$, no uterine cervix, the right side of the round ligament within normal limits, the rightside tube with the right side hematosalping measuring $7 \times 5 \times 3 \mathrm{~cm} 3$, the rightside ovary measuring $3 \times 3 \times 2 \mathrm{~cm} 3$. Left hemiuterus size $3 \times 3 \times 2 \mathrm{~cm} 3$, not functioning, left side rotundum ligament within normal limits, left tube within normal limits, left ovary $2 \times 2 \times 2 \mathrm{~cm} 3$. There is a connective tissue that connects the inferior side of the right and left hemiuterus. Vulva with hymen within normal limits, not bulging. Vagina, the depth of the vagina is $9 \mathrm{~cm}$. On the right side of the vagina is the inferior portion of the right hemiuterus with prominent hematometra, palpable cystic, without uterine cervix.

From the results of the examination and surgery, it was found that the ESHRE classification was U4a not communicating, C4 aplasia, V0 normal. Classification of HWW syndrome, namely classification 1.2 .

The surgical procedures performed in this patient were vaginal drainage of the right hemiuterus hematometra and right hematosalping. Bleeding during surgery, including hematometric fluid and hematosalping amounted to $100 \mathrm{ml}$.

Postoperative evaluation was obtained regular menstruation every month, about 7 days, no pain, no other complaints. Defecate and urinate within normal limits. On examination of vaginal introitus within normal limits, there was no infection, no signs of inflammation, the vagina was intact, there were no areas of adhesions Figure 2.

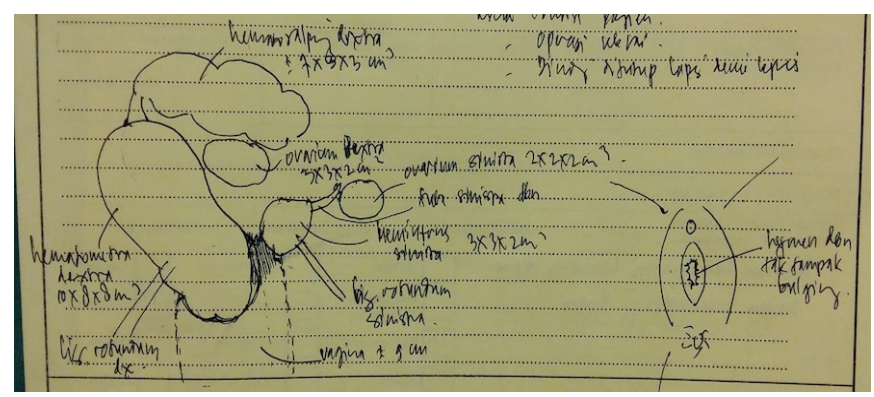

Figure 2 Image of case I genital organ illustration.

\section{Case 2}

Case 2 was a 12 year old, P0A0, referral from a private hospital with a history of hymen incisions and vaginal introitus. The main complaint of the patient is abdominal pain, never having menstruation.

From the examination, the data shows that the stomach is flat, supple, painless, the uterus is enlarged as big as an adult's fist. Urethra and vulva within normal limits, visible hymen incision signs. From a rectal examination, the uterus is enlarged to a distance of $4 \mathrm{~cm}$ proximal to the anus. On ultrasound examination of the didelphys uterus, the size of the right hemiuterus was $7 \times 6,2 \times 5,7 \mathrm{~cm} 3$ and the left hemiuterus was $6,3 \times 5,2 \times 4 \mathrm{~cm} 3$, the overall width of the uterus was $12,65 \mathrm{~cm}$. There is a channel between the right and left hemiuterus in the cervical area with a width of $2,8 \mathrm{~cm}$, there is a blood flow of hematometra in this area, the left kidney is visible while the right kidney is not.
From the MSCT examination of the abdomen, it was found that there was a Mullerian duct anomaly, uterus didelphys with hematometra and double vagina, left hematocolpos due to imperforate hymen accompanied by bilateral hydrosalping. There is agenesis of the right kidney, left hydroureter. Lumbosacral unstable. From abdominal radiograph examination and intravenous pyelography, it shows a picture of delayed right kidney function, disruption of the left ureteral tract, opacity in the pelvic cavity with suspicion of originating from a mass in the pelvic cavity.

This patient was diagnosed with hematometra, vaginal agenesis, history of hymen incision, suspected HWW syndrome. It was decided that the surgical procedure was carried out together with the urologist and digestive surgeon.

At the time of surgery, data on the size of the left and right didelphys uterus were obtained $9 \times 4 \times 3 \mathrm{~cm} 3$, after opening, the depth of the right and left uterine cavity sonde was $9 \mathrm{~cm}$ each. The right and left ovaries grew by $5 \times 4 \times 3 \mathrm{~cm} 3$. The right and left tubes were enlarged with bilateral hematosalping, each measuring $4 \times 2 \times 2 \mathrm{~cm} 3$. According to the ESHRE classification, the patient's diagnosis can be grouped as U4a communication, C4 aplasia, V4 aplasia. Based on HWW syndrome classification, including classification 2.2.

The surgical procedure performed is cystoscopy performed by a urologist. The mouth of the right ureter was not identified. The mouth of the left ureter is at the neck of the bladder (ectopic). Left ureter catheter was inserted and bladder exploration indicated for agenesis of the right kidney and left ectopic ureter.

The surgeries performed by gynecologists and digestive surgeons are adhesiolytic laparotomy, end-to-end sigmoid anastomosis sigmoid colon resection, sigmoid vaginoplasty (isoperistaltic), end-to-side uterosigmoid anastomosis (on the posterocudal wall of the uterine body, longitudinal incision of the didelphys uterine, the incision direction based on the midline, incision length $3 \mathrm{~cm}$ ), bilateral cystectomy, bilateral meatotomy, indicated by a didelphys uterus, bilateral choccolate cyst, bilateral hematosalping, with adhesions, in HWW syndrome. The amount of bleeding during surgery is about $1000 \mathrm{ml}$.

Post-operative evaluation found regular menstruation every month, approximately 7 days, no pain, no complaints. Defecate and urinate within normal limits. On examination of vaginal introitus 1,5 $\mathrm{cm}$ in diameter with good wound edges, no infection, no signs of inflammation, intact vagina, no adhesions Figure 3, Table 1.

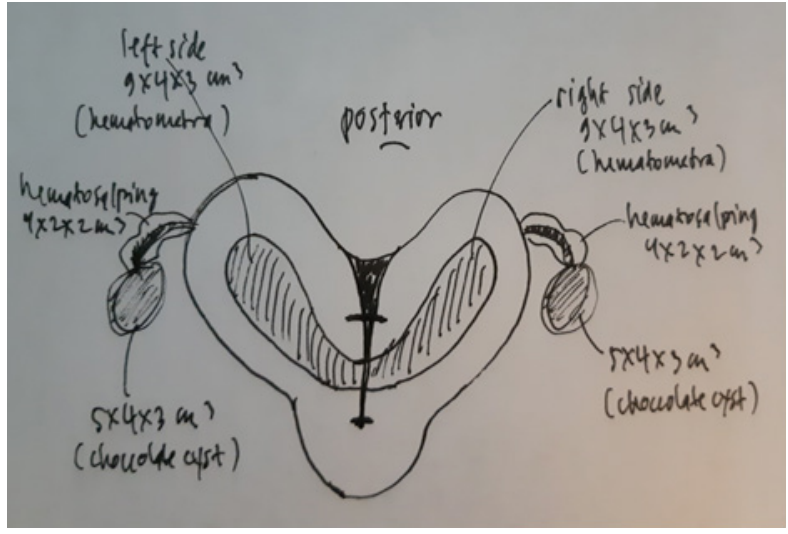

Figure 3 Image of case 2 genital organ illustration. 
Table I Comparison between the Two Cases

\begin{tabular}{|c|c|c|}
\hline Data & Case I & Case 2 \\
\hline Age & 10 years old & 12 years old \\
\hline Main complaints & abdominal pain, never had a period & abdominal pain, never had a period \\
\hline \multirow[t]{2}{*}{$\begin{array}{l}\text { HWW syndrome } \\
\text { classification }\end{array}$} & I. Completely obstructed hemivagina & 2. Incompletely obstructed hemivagina \\
\hline & I.2. Cervicovaginal atresia without communicating uteri & 2.2. With communicating uteri \\
\hline ESHRE classification & U4a non-communicating/not horn, C4 aplasia, V0 normal, & U4a communicating, C4 aplasia, V4 aplasia \\
\hline Uterus & $\begin{array}{l}\text { Right hemiuterus with hematometra, rudimenter left } \\
\text { hemiuterus }\end{array}$ & Didelphys uterus, hematometra \\
\hline Cervical uterus & Not formed & $\begin{array}{l}\text { There is a connection in the cervical area of the uterus didelphys } \\
\text { (communicate) }\end{array}$ \\
\hline Salping & Right hematosalping, left salping in a normal condition & Bilateral hematosalping \\
\hline Ovarium & Normal & Bilateral chocolate cyst \\
\hline Endometriosis & There is no endometriosis & There is adhesion, endometriosis \\
\hline Vagina & Within normal limits & There is no vagina \\
\hline Kidney & Agenesis of right kidney & Agenesis of right kidney \\
\hline Ureter & Normal left ureter, agenesis right ureter & $\begin{array}{l}\text { Left hydroureter, ectopic left ureter, estuary left ureter in bladder } \\
\text { neck, agenesis right ureter }\end{array}$ \\
\hline Bladder, urethra & Normal & Normal \\
\hline
\end{tabular}

\section{Discussion}

In both cases reported, the main complaints were the same abdominal pain, and had never had a period. Case 1 is younger at 10 years old, while case 2 is 12 years old. This is consistent with the theory that HWW syndrome is usually found at puberty, with symptoms that are not very specific. A history of analgesic drug administration in both cases also slowed down the detection of abnormalities. On further examination it was found that the pain was caused by hematometra and hematosalping which occurred in both cases. Case 1 had hematometra in the right hemiuterus and right hematosalping, whereas case 2 had hematometra in the uterus didelphis and bilateral hematosalping.

There is an abnormality in the urinary tract in the form of agenesis of the right kidney. This is also in accordance with the theory that renal agenesis occurs more ipsilaterally, and abnormalities occur more frequently on the right side (both cases of agenesis are in the right kidney). The left kidney in both cases was normal, with the left hydroureter and ectopic ureter at the neck of the bladder for case 2 . There is no malposition of the ovary or intestinal malrotation.

Surgical therapy is carried out with the main objective of draining menstrual blood from the hematometra and hematosalping. In case 1, where the vagina is within the normal length range $(9 \mathrm{~cm}$ long) and the area of the right hemiuterus inferiorly protrudes toward the vagina, an area that can be made with a drainage incision can be identified to remove all of the hematometra and hematosalping. The area where the circular incision is performed forms a hole with a diameter of $1,5 \mathrm{~cm}$. Installation of foley catheter number 24F which is maintained for 2 weeks, to keep the hole that has been made permanent and not closed for the long term.

Second case, patient with vaginal agenesis. The incidence of vaginal agenesis varies from 1 in 4,000 to 1 in 80,000 young women. A congenital abnormality in the form of a vaginal absence is usually first seen by an obstetrician at around 14 years of age. Patient came to have her checked out because she was worried that menstruation had not yet occurred until that age. ${ }^{8}$

In young female patients with vaginal agenesis who have a functional uterus, neovaginal preparations should be done immediately to treat the hematometra condition resulting in pelvic pain. It should be noted that this attempt to construct a vaginal or neovaginal tract should function to drain all menstrual blood throughout the patient's life. However, this patient is still a child so it is unwise for the patient to undergo continuous vaginal dilatation after surgery to maintain patency of the vaginal canal. Then, it was decided to do a vaginoplasty surgery using the sigmoid colon. This surgical technique does not require post-surgical dilatation because the diameter of the neovaginal hole is sufficient to drain menstrual blood.

The area that is prepared to become a neovagina is made by sharply opening the rectovesical cavity, through the vaginal introitus, deepening and widening it to reach the peritoneal cavity. A $15 \mathrm{~cm}$ long sigmoid graft colon was prepared, maintaining the vascularization of the sigmoid artery.

Colonic anastomosis with vaginal introitus may be performed with interrupted absorbable sutures. This procedure is performed by first exposing the peritoneum to the soft dimple with a sharp dissection using a guide that is inserted through the perineo-abdominal tunnel that has been created. ${ }^{8}$ In this $2^{\text {nd }}$ case patient, the sigmoid colon graft is implanted through a rectovesical space which is made about 3 centimeters in diameter. The proximal end of the sigmoid graft is implanted into the inferior posterior side of the didelphys uterus (where the uterine cervix fuses) with 2 layers of end-to-side sutures. The distal end of the sigmoid graft is sutured into the vagina introitus with interupted sutures.

Post-surgical evaluations are carried out weekly for 2 weeks, monthly, up to every 6 months to one year. Surgical wound healing is 
good. In case 1, the hole on the inferior side of the right hemiuterus was still patent and did not close. In case 2 , The neovagina originating from a sigmoid colon graft can be well implanted, fusing with the vaginal introitus. Sigmoid vaginoplasty seems to be the right procedure for case number 2 . There is no infection. Henceforth, the function of the genital tract can be well known when the patient has vaginal menstruation after surgery. ${ }^{8}$

Neovaginal stenosis was the major long-term complication in all intestinal segments $(2,9 \%$ jejunum, $5,4 \%$ ileum, $6,1 \%$ cecum and $8,8 \%$ sigmoid) in the first postoperative year. This can be treated with dilation or surgical intervention. Daily use of vaginal dilators for several weeks postoperatively can prevent neovaginal stenosis. ${ }^{9}$ Case number 2 had mild stenosis that occurred in vaginal introitus from $3 \mathrm{~cm}$ to about $1,5 \mathrm{~cm}$ in size, a condition within reasonable limits that is also found in other case reports. ${ }^{10}$ No dilation is done because the diameter is enough to drain menstrual blood every month. Both patients can get menstruation every month, 6-7 days, no pain, no complaints.

Reconstructive surgery to create a vagina with intestinal vaginoplasty is a surgery that requires high skills. This action can produce a neovagina that can function in the long term, with good lubrication. It can be argued that the utero-coloneovaginoplasty is a safe procedure for maintaining menstrual blood flow in patients with a functional uterus. ${ }^{11}$

It is expected that the results of surgery can function properly for the entire patient's lifetime. If the patient is more mature and has married so that there is sexual activity, in case 2 , it is necessary to prepare with vaginal dilatation first to dilate the vaginal introitus if necessary. The occurrence of pregnancy is still possible, but labor must be done in a planned surgery.

It is often said that excessive vaginal discharge is a problem in the majority of patients undergoing intestinal vaginoplasty. This discharge is temporary and generally decreases significantly in 3-6 months postoperatively until it is no longer a matter of concern for the patient. Reported only $0.2 \%$ of cases with persistent vaginal discharge. Neovaginal odor was reported in $1,2 \%$ of cases of vaginoplasty with sigmoid segments, but was not mentioned in studies of vaginoplasty with jejunal, ileum and cecum segments. ${ }^{9}$ In this case, there was no excessive discharge or foul odor that interfered with the patient's daily life. ${ }^{12}$

\section{Conclusion}

The clinical manifestations of Herlyn-Werner-Wunderlich syndrome (HWW) cases can be classified into 2 groups, complete and incomplete. Surgery is done based on the condition of the uterus and vagina. In the case of uterine cervical agenesis, but with a normal vagina, surgery for uterine cervical tissue drainage can be performed. If vaginal agenesis is found, vaginoplasty can be performed and one option is a sigmoid colon vaginoplasty.

\section{Acknowledgments}

Part of this manuscript was presented at an International Conference on Obstetrics, Gynecology \& Pediatrics event, November 26-28, 2018, Kuala Lumpur Malaysia.

\section{Conflicts of interest}

The author declares there is no conflict of interest.

\section{References}

1. Gholoum S, Puligandla PS, Hui T, et al. Management and outcome of patients with combined vaginal septum, bifid uterus, and ipsilateral renal agenesis (Herlyn-Werner-Wunderlich syndrome). Journal of pediatric surgery. 2006;41(5):987-992.

2. Acién P, Acién M, Sánchez-Ferrer M. Complex malformations of the female genital tract. New types and revision of classification. Human reproduction (Oxford, England). 2004;19(10):2377-2384.

3. Orazi C, Lucchetti MC, Schingo PM, et al. Herlyn-Werner-Wunderlich syndrome: uterus didelphys, blind hemivagina and ipsilateral renal agenesis. Sonographic and MR findings in 11 cases. Pediatric radiology. 2007;37(7):657-665.

4. David A, Gudi SN, Shankar R. Herlyn-Werner-Wunderlich syndrome: Premenarche. Journal of SAFOG. 2017;9(2):207-210.

5. Khaladkar SM, Kamal V, Kamal A, et al. The Herlyn-Werner-Wunderlich Syndrome - A Case Report with Radiological Review. Polish journal of radiology. 2016;81:395-400.

6. Zhu L, Chen N, Tong JL, et al. New classification of Herlyn-WernerWunderlich syndrome. Chinese medical journal. 2018;128(2):222-225.

7. Vercellini P, Daguati R, Somigliana E, et al. Asymmetric lateral distribution of obstructed hemivagina and renal agenesis in women with uterus didelphys: institutional case series and a systematic literature review. Fertility and sterility. 2007;87(4):719-724.

8. Cam C, Celik C, Sakalli M, et al. Introito-cervical sigmoid colon transpositioning procedure. Cent Eur J Med. 2010;5(2):184-188.

9. Robbens C, Monstrey S. Intestinal Vaginoplasty: Which should be the bowel segment of choice? Dissertation presented in the $2^{\text {nd }}$ Master year in the programme of Master of Medicine in Medicine, Ghent University. 2015.

10. Karateke A, Gurbuz A, Haliloglu B, et al. Intestinal vaginoplasty: is it optimal treatment of vaginal agenesis? A pilot study. Surgical method of sigmoid colon vaginoplasty in vaginal agenesis. International urogynecology journal and pelvic floor dysfunction. 2006;17(1):40-45.

11. Kisku S, Varghese L, Kekre A, et al. Bowel vaginoplasty in children and young women: an institutional experience with 55 patients. International urogynecology journal. 2015;26(10):1441-1448.

12. Committee on Adolescent Health Care. ACOG Committee Opinion No. 728: Müllerian Agenesis: Diagnosis, Management, And Treatment. Obstetrics and gynecology. 2018;131(1):e35-e42. 